\title{
User-Centred Design of Augmented and Virtual Reality Human- Computer Interfaces
}

\author{
Dr. Roy Eagleson \\ University of Western Ontario, Canada
}

AR/VR is like a double-edged sword: Displays can be compelling and powerful - yet when certain design principles are violated, they can be confusing and unusable. We explore some of the reasons for this, from the perspective of HumanComputer Interaction, which necessitates a design process that is informed by basic scientific results from the overlapping domains of Perception, Action, and Cognition.

This keynote lecture will explore the special "capacities and constraints" of these three basic subsystems. The talk will then examine some of the implications and fundamental concerns for general AR. The talk will focus on the implications for particular applications in various Multimedia applications, and some which are specialized for Surgical Planning, Guidance, and targeting, with case studies drawn from published papers on the use of AR for Neurosurgery. 